\title{
Fertilizantes agrícolas aplicados via solução nutritiva em Cattleya labiata Lindl. ${ }^{(1)}$
}

\author{
RODRIGO THIBES HOSHINO(2)*; GUILHERME AUGUSTO CITO ALVES(2); RENAN RIBEIRO BARZAN(2); GUSTAVO \\ ADOLFO DE FREITAS FREGONEZI ${ }^{(3)}$ e RICARDO TADEU DE FARIA(2)
}

\begin{abstract}
RESUMO
A fertilização via solução nutritiva é comumente utilizada na produção comercial de flores em vaso, sendo empregadas formulações solúveis a base de NPK. Entretanto, a maioria das formulações comerciais disponíveis no mercado apresentam custos elevados e a utilização de fertilizantes agrícolas pode ser uma alternativa economicamente viável. O objetivo deste trabalho foi avaliar a eficácia de fertilizantes agrícolas, aplicados via solução nutritiva, na substituição de formulações comerciais, no crescimento inicial de Cattleya labiata. Mudas de C. labiata propagadas in vitro foram cultivadas em casa de vegetação com temperatura controlada, $27 \pm$ $3^{\circ} \mathrm{C}$, e retenção de $50 \%$ da luminosidade. Foram utilizados vasos de polipropileno com capacidade de $0,415 \mathrm{~L}$, contendo mistura de casca de pinus e carvão (1:1, v:v), como substrato. Ao todo foram utilizados 4 tratamentos, sendo: Sem fertilização (Testemunha); Peters $^{\circledR}$ - NPK $202020\left(3 \mathrm{~g} \mathrm{~L}^{-1}\right)$; NPK - ureia $\left(1,3 \mathrm{~g} \mathrm{~L}^{-1}\right)$, superfosfatos simples $\left(3,3 \mathrm{~g} \mathrm{~L}^{-1}\right)$ e cloreto de potássio $\left(1,0 \mathrm{~g} \mathrm{~L}^{-1}\right)$; $\mathrm{NK}_{\text {- }}$ ureia $\left(1,3 \mathrm{~g} \mathrm{~L}^{-1}\right)$ e de cloreto de potássio $\left(1,0 \mathrm{~g} \mathrm{~L}^{-1}\right)$. Foram aplicados $50 \mathrm{~mL}$ da solução nutritiva por vaso quinzenalmente de acordo com os tratamentos. Foram avaliadas características fitométricas, $\mathrm{pH}$ e a condutividade elétrica do substrato além dos teores e o acúmulo de macronutrientes. $\mathrm{O}$ delineamento experimental foi inteiramente casualizado com 12 repetições, sendo considerado cada vaso com duas plantas como uma unidade experimental. Os parâmetros foram submetidos à análise de variância e teste de Tukey a 5\% de probabilidade. Em relação aos parâmetros fitométricos não foram observadas diferenças significativas entre os tratamentos que receberam fertilizações, porém os mesmos foram superiores à testemunha em altura e massa seca de parte aérea. Os nutrientes mais acumulados foram o $\mathrm{K}>\mathrm{Ca}>\mathrm{N}$, nos tratamentos que receberam fertilizações. $\mathrm{O}$ uso de ureia, superfosfato simples e cloreto de potássio, aplicados em solução nutritiva, apresentam potencial para substituir as formulações comerciais no crescimento inicial da orquídea nativa Cattleya labiata.
\end{abstract}

Palavras-chave: Orchidaceae, floricultura, fertirrigação.

\begin{abstract}
Agricultural fertilizers applied as nutrient solution in Cattleya labiata Lindl.

Fertilization through nutrient solution is commonly used in commercial production of potted flowers, being employed soluble NPK formulations. However, most commercial formulations available in the market have high costs and the use of agricultural fertilizers can be an economically viable alternative. The objective of this study was to evaluate the effectiveness of agricultural fertilizers applied as nutrient solutions, in replacement of commercial formulations, on initial growth of Cattleya labiata. C. labiata seedlings in vitro propagated were grown in a greenhouse with controlled temperature, $27 \pm 2{ }^{\circ} \mathrm{C}$, and retaining $50 \%$ brightness. Polypropylene pots were used with $0.415 \mathrm{~L}$ capacity, containing a mixture of pine bark and charcoal (1:1, v:v) as substrate. Altogether were used 4 treatments: without fertilization (control); Peters ${ }^{\circledR}$ - NPK $202020\left(3.0 \mathrm{~g} \mathrm{~L}^{-1}\right)$; NPK - urea $\left(1.3 \mathrm{~g} \mathrm{~L}^{-1}\right)$, superphosphate $\left(3.3 \mathrm{~g} \mathrm{~L}^{-1}\right)$ and potassium chloride $\left(1.0 \mathrm{~g} \mathrm{~L}^{-1}\right)$; $\mathrm{NK}$ - urea $\left(1.3 \mathrm{~g} \mathrm{~L}^{-1}\right)$ and potassium chloride $\left(1.0 \mathrm{~g} \mathrm{~L}^{-1}\right)$. The nutrient solutions were applied 50 $\mathrm{ml}$ per pot every two weeks, according to the treatments. Were evaluated phytometric characteristics, $\mathrm{pH}$ and electrical conductivity of the substrate beyond the levels and accumulation of macronutrients. The experimental design was completely randomized with 12 replications, being considered each pot with two plants as an experimental unit. The parameters were submitted to ANOVA and Tukey's test at 5\% probability. Regarding phytometric parameters no significant differences were observed between the treatments that received fertilization, which are superior to the control in height and dry mass of shoots. The more accumulated nutrients were $\mathrm{K}>\mathrm{Ca}>\mathrm{N}$, in the treatments that received fertilization. The use of urea, superphosphate and potassium chloride, applied in nutrient solution, have potential to replace the commercial formulations in the initial growth of native orchid Cattleya labiata.
\end{abstract}

Keywords: Orchidaceae, floriculture, fertigation.

\section{INTRODUÇÃO}

A produção do país em flores e plantas ornamentais vem aumentando com o passar dos anos, em todas as regiões do Brasil, sendo o mercado interno o principal consumidor. No Brasil, a cadeia produtiva de flores e plantas ornamentais, em 2013, movimentou R \$ 5,22 bilhões, na qual o segmento de flores envasadas, representou $24,12 \%$ da movimentação financeira, sendo as orquídeas destaque devido ao seu desempenho produtivo e comercial (JUNQUEIRA e PEETZ, 2014).

Segundo Cardoso et al. (2016), as orquídeas do gênero Cattleya e seus híbridos são altamente valorizadas no mercado, podendo competir com os gêneros Phalaenopsis

\footnotetext{
(1) Recebido em 01/03/2016 e aprovado em 16/09/2016

(2) Universidade Estadual de Londrina (UEL), Londrina-PR, Brasil. *Autor correspondente: rodrigohoshino@gmail.com

${ }^{(3)}$ Centro Universitário Filadélfia (UNIFIL), Londrina-PR, Brasil.
} 
e Dendrobium. A Cattleya labiata Lindl. é uma orquídea nativa brasileira muito utilizada no melhoramento e produção de híbridos comerciais.

Dentre os manejos para produção comercial de ornamentais, a fertilização é essencial, pois promove ganhos na qualidade, padronização e redução no tempo de cultivo, onde a determinação das exigências nutricionais para cada espécie é uma importante estratégia para aumentar a eficiência de produção. A maioria dos fertilizantes existentes no mercado não foi desenvolvida considerando a especificidade de cada espécie, acarretando em baixa qualidade e elevado custo de produção, justificando a importância dos estudos nutricional para cada espécie (NETO et al., 2015).

Neste âmbito pode-se destacar o uso de formulações solúveis, usualmente aplicadas via irrigação, a qual apresenta vantagens como concentração e frequência, facilmente alteradas conforme as exigências do cultivo, sendo sua utilização sistematizada de acordo com a infraestrutura das casas de vegetação por meio da irrigação. Entretanto o uso de fórmulas comerciais pode onerar os custos de produção, onde o uso de produtos agrícolas como ureia, cloreto de potássio e superfosfato simples, pode ser uma alternativa simples, econômica e de fácil aquisição.

É reconhecido que o habitat epifítico possui baixa disponibilidade de nutrientes, no qual as plantas epífitas apresentam eficiente capacidade de absorção e remobilização de nutrientes (ZOTZ, 2004). Entretanto em cultivos comerciais as fontes naturais de nutrientes ficam comprometidas, Naik et al. (2009), destacam em orquídeas epífitas, que os nutriente N, P e K são os mais estudados na fertilização inorgânica. Bichsel et al. (2008), concluem que a utilização de N, P e K aplicados via solução nutritiva, favorecem o crescimento vegetativo e desenvolvimento reprodutivo em Dendrobium sp.

O objetivo deste trabalho foi avaliar a eficácia de fertilizantes agrícolas, aplicados via solução nutritiva, na substituição de formulações comerciais, no crescimento inicial de Cattleya labiata.

\section{MATERIAL E MÉTODOS}

O ensaio foi conduzido na Universidade Estadual de Londrina, localizada na, longitude $51^{\circ} 11^{\prime}$ oeste e latitude $23^{\circ} 23^{\prime}$ sul, com altitude de $566 \mathrm{~m}$, entre os meses de setembro de 2013 a maio de 2014 , totalizando 240 dias. O clima da região é classificado como subtropical úmido (Cfa), segundo Kopen. O experimento permaneceu em cultivo protegido dentro de casa de vegetação, coberta com placas de policarbonato transparente e sistema de resfriamento humid cool, com temperatura controlada a $27 \pm 3^{\circ} \mathrm{C}$ e $50 \%$ de retenção solar pela utilização de

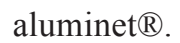

Foram utilizadas mudas aclimatizadas, com 10 meses de idade ex vitro, da orquídea Cattleya labiata (Lindl.), provenientes da semeadura in vitro, as quais no momento da instalação do experimento apresentavam aproximadamente $5,5 \mathrm{~cm}$ de altura. As plantas foram transplantadas para vasos de polipropileno de coloração preta com capacidade de $0,415 \mathrm{~L}$, e no fundo de cada vaso foi adicionada uma camada de cacos cerâmicos para auxiliar a drenagem. A irrigação foi manual, realizada diariamente. Como substrato foi utilizado a mistura de casca de pinus e carvão, na proporção de 1:1 (v:v), peneirados entre peneiras com crivo de 1,5 e $0,5 \mathrm{~cm}$.

Foram aplicados quatro tratamentos sendo: Sem fertilização (Testemunha); Peters ${ }^{\circledR}$ - fertilização com Peters ${ }^{\circledR}$ (NPK 2020 20); NPK - fertilização com ureia, superfosfato simples e cloreto de potássio; NK - fertilização com ureia e cloreto de potássio (sem fornecimento de fósforo via solução nutritiva). As soluções nutritivas foram preparadas com concentrações de nutriente equivalentes a $600 \mathrm{mg} \mathrm{L}^{-1}$ de $\mathrm{N}, 262 \mathrm{mg} \mathrm{L}^{-1}$ de $\mathrm{P}$ e $498 \mathrm{mg} \mathrm{L}^{-1}$ de $\mathrm{K}$, equivalentes as concentrações disponibilizadas pelo fertilizante comercial Peters $^{\circledR}$ (NPK 2020 20) na dosagem de 3,0 $\mathrm{g} \mathrm{L}^{-1}$. O delineamento experimental foi inteiramente casualizado com 12 repetições, sendo considerado cada vaso com duas plantas como uma unidade experimental.

O fertilizante solúvel Peters ${ }^{\circledR}$ na formulação NPK 20 20 20, com $4.194 \mathrm{~g} \mathrm{~L}^{-1}$ de solubilidade, produzido pela empresa Everris-EUA, contém as seguintes garantias: nitrogênio (20\%), sendo, nitrogênio amoniacal (4,1\%), nitrogênio nítrico $(5,5 \%)$ e ureia $(10,4 \%), \mathrm{P}_{2} \mathrm{O}_{5}(20 \%)$, $\mathrm{K}_{2} \mathrm{O}(20 \%)$, boro $(0,0125 \%)$, cobre $(0,0125 \%)$, ferro $(0,5 \%)$, manganês $(0,025 \%)$, molibdênio $(0,005 \%)$ e zinco $(0,025 \%)$. Derivado dos fertilizantes: fosfato de amônio, nitrato de potássio, ureia, fosfato de potássio, sulfato de magnésio, ácido bórico, cobre EDTA, ferro EDTA, manganês EDTA, molibdato de amônio e zinco EDTA.

Para os fertilizantes agrícolas foram utilizados 1,3 $\mathrm{g}$ $\mathrm{L}^{-1}$ de ureia ( $45 \%$ de $\mathrm{N}$ ) com $1.000 \mathrm{~g} \mathrm{~L}^{-1}$ de solubilidade, $3,3 \mathrm{~g} \mathrm{~L}^{-1}$ de superfosfato simples $\left(18 \% \mathrm{P}_{2} \mathrm{O}_{5}\right)$ com $20 \mathrm{~g} \mathrm{~L}^{-1}$ de solubilidade e $1,0 \mathrm{~g} \mathrm{~L}^{-1}$ de cloreto de potássio $(60 \%$ de $\mathrm{K}_{2} \mathrm{O}$ ) com $340 \mathrm{~g} \mathrm{~L}^{-1}$ de solubilidade. O tratamento contendo superfosfato simples foi filtrado após a moagem e diluição do referido fertilizante. As soluções foram aplicadas a cada 15 dias, adicionando $50 \mathrm{~mL}$ das soluções por vaso e no tratamento sem adubação foi aplicado água potável.

Foram avaliadas após 240 dias do inicio da aplicação dos tratamentos as seguintes características fitométricas da parte aérea: massa seca da parte aérea (MSPA), altura da parte aérea (ALT), comprimento do pseudobulbo (CPB), diâmetro do pseudobulbo (DPB) e número de brotos. Do sistema radicular foram avaliados: massa seca de raízes (MSR), comprimento médio das raízes principais (CMR) e número de raízes. Do substrato foram avaliados o $\mathrm{pH}$ e condutividade elétrica (KÄMPF, 2006).

As avaliações das características: altura da parte aérea, comprimento e diâmetro do pseudobulbo foram realizados no maior pseudobulbo com folha completamente expandida. Foram considerados como brotos, estruturas que não apresentavam pseudobulbos formados e folhas expandidas. O comprimento médio das raízes principais foi realizado por mensuração do comprimento de todas as raízes principais.

Para quantificação da massa seca, os tecidos da parte aérea e raízes foram lavados primeiramente em água corrente e posteriormente em água deionizada, após as 
lavagens os materiais foram secos em estufa de ventilação forçada a $55^{\circ} \mathrm{C}$, até atingirem peso constante. Após secos e pesados os tecidos da parte aérea, foram moídos em moinho analítico, para posterior determinação das concentrações dos seguintes nutrientes: nitrogênio $(\mathrm{N})$, fósforo $(\mathrm{P})$, potássio $(\mathrm{K})$, cálcio $(\mathrm{Ca})$ e magnésio $(\mathrm{Mg})$, segundo a metodologia proposta por (MALAVOLTA et al., 1997).

Os parâmetros foram submetidos à análise de variância e teste de Tukey a 5\% de probabilidade, através do software estatístico Sisvar (FERREIRA, 2011).

\section{RESULTADOS E DISCUSSÃO}

Foi possível observar que, de modo geral, independente da fertilização utilizada, (Peters ${ }^{\circledR}$, NPK ou NK agrícolas) o desenvolvimento fitométrico da parte aérea apresentou uma resposta similar nos tratamentos, os quais foram superiores à testemunha, com incrementos de massa seca e altura (Tabela 1). Estes resultados indicam uma possível alternativa à utilização de formulações solúveis comerciais por fontes agrícolas.

Tabela 1. Desenvolvimento fitométrico na parte aérea da Cattleya labiata. Massa seca da parte aérea (MSPA), altura (ALT), comprimento de pseudobulbo (CPB), diâmetro do pseudobulbo (DPB) e número de brotos (Broto).

Table 1. Development of aerial parte in Cattleya labiata. Shoot dry mass (MSPA), height (ALT), length of pseudobulb $(C P B)$, diameter of the pseudobulbs (DPB) and number of sprouts (Broto).

\begin{tabular}{|c|c|c|c|c|c|c|c|c|c|c|}
\hline \multirow[b]{2}{*}{ Trat } & \multicolumn{10}{|c|}{ Parte Aérea } \\
\hline & $\begin{array}{c}\text { MSPA } \\
\text { (mg) }\end{array}$ & & $\begin{array}{l}\text { ALT } \\
(\mathbf{c m})\end{array}$ & & $\begin{array}{l}\text { CPB } \\
(\mathrm{cm})\end{array}$ & & $\begin{array}{c}\text { DPB } \\
(\mathrm{mm})\end{array}$ & & $\begin{array}{c}\text { Broto } \\
\left(\mathbf{n}^{\circ}\right)\end{array}$ & \\
\hline Test & 1413 & $b^{1}$ & 8,9 & $\mathrm{~b}$ & 3,0 & $\mathrm{~b}$ & 8,04 & $\mathrm{bc}$ & 0,4 & a \\
\hline Peters & 2368 & $\mathrm{a}$ & 11,5 & $\mathrm{a}$ & 3,8 & $a b$ & 9,81 & $a b$ & 1,3 & a \\
\hline NPK & 2593 & a & 11,6 & a & 4,3 & $\mathrm{a}$ & 6,84 & c & 1,2 & a \\
\hline NK & 2507 & a & 11,5 & $\mathrm{a}$ & 3,9 & $a b$ & 10,58 & $\mathrm{a}$ & 1,3 & $\mathrm{a}$ \\
\hline $\mathrm{CV}(\%)$ & 23,00 & & 18,90 & & 26,10 & & 10,58 & & $23,13^{2}$ & \\
\hline
\end{tabular}

${ }^{1}$ Médias seguidas da mesma letra não diferem pelo teste de Tukey a 5\%. Trat: tratamentos. Test: testemunha. Peters: Peters 20:20:20. NPK: ureia + cloreto de potássio + superfosfato simples. NK: ureia + cloreto de potássio. ${ }^{2}$ Dados transformados $(\sqrt{\mathrm{x}}+1) .{ }^{1}$ Means followed by same letter don t differ by 5\% Tukey test. Trat: treatments. Test: control. Peters: Peters 2020 20. NPK: urea + potassium chloride + superphosphate. NK: urea + potassium chloride. ${ }^{2}$ Transformed data $(\sqrt{ } x+1)$.

Uma das principais razões nos ganhos da parte aérea é atribuída a expansão e desenvolvimento das folhas, possivelmente estimulado pelo fornecimento dos nutrientes, principalmente de $\mathrm{N}$, que resultou em plantas mais altas e consequentemente refletiu no acúmulo de massa seca. Zong-min et al. (2012) relataram que a aplicação de N em Paphiopedilum armeniacum, resultou em incrementos da área e comprimento foliar. Para Dendrobium nobile, o aumento da concentração de $\mathrm{N}$ em solução, de 0 para 100 $\mathrm{mg} \mathrm{L}^{-1}$, houve um incremento de $76 \%$ em altura (BICHSEL et al., 2008). Deste modo as fertilizações, contribuíram no desenvolvimento das mudas, uma vez que, no crescimento inicial vegetativo de folhas e raízes recém-formadas, há alta demanda por nutrientes (SUSILO et al. 2013).

Apesar das conhecidas funções do $\mathrm{N}$, os resultados podem variar com o hábito de crescimento, estágio de desenvolvimento e a relação entre nitrato e amônio, pois de acordo com Pan et al. (1997) a forma nítrica é preferencialmente absorvida por Cymbidium sinense, (orquídea terrícola). Entretanto orquídeas epífitas se desenvolvem melhor quando adubadas com fontes amoniacais em detrimento das fontes nítricas. Além disso, estudos in vitro indicam que a ureia pode ser absorvida diretamente pelas raízes, sem necessidade de hidrólise prévia, em que este adubo e o amônio são as formas preferenciais de $\mathrm{N}$ absorvidas (TRÉPANIER et al., 2009). Wang e Konow (2002) em estudo com Phalaenopsis obtiveram plantas com massa fresca de folhas 50\% superior e área foliar $40 \%$ maior, quando fertilizantes que continham ureia em sua composição foram aplicados, em relação à fertilização com outras fontes de $\mathrm{N}$.

Para os demais parâmetros da parte aérea avaliados, nenhuma das fertilizações se destacou. O tratamento NPK agrícola foi superior à testemunha em relação ao comprimento do pseudobulbo, entretanto possibilitou o menor diâmetro do mesmo. Por sua vez o diâmetro do pseudobulbo no tratamento $\mathrm{NK}$ agrícola foi o único a diferir do tratamento sem fertilização.

Os pseudobulbos constituem uma importante estrutura de reserva de água e nutrientes (NG e HEW, 2000), contudo não houve resposta da planta às fertilizações em relação a esse parâmetro, ocorrendo de modo similar com número de brotos, nos quais não se observaram diferenças estatísticas entre os tratamentos. Porém em Dendrobium, plantas fertilizadas com soluções deficientes em P e K apresentaram redução nos pseudobulbos (BICHSEL et al., 2008), e em Cymbidium características como comprimento e espessura do pseudobulbo foram aumentadas com a aplicação de diferentes formulações de NPK, tendo apresentado também maior número dos mesmos (NAIK et al, 2013). Estes resultados divergentes podem ser atribuídos ao estádio inicial das plantas de Cattleya, utilizadas no presente estudo, onde nos trabalhos citados foram utilizadas plantas em florescimento, além das diferenças intergenéricas. 
Para o sistema radicular, apesar do comprimento médio das raízes principais, nos tratamentos contendo fontes agrícolas, apresentarem redução em relação à testemunha, não foram observadas diferenças estatísticas em acúmulo de massa seca. Isto ocorreu devido à compensação pelo incremento no número de raízes, onde o tratamento NPK agrícola se apresentou significativamente superior ao tratamento testemunha (sem adubação) (Tabela 2).

Tabela 2. Desenvolvimento fitométrico do sistema radicular da Cattleya labiata e caracterização química do substrato. Massa seca de raízes (MSR), comprimento médio das raízes principais (CMR), número de raízes (Raízes), pH do substrato $(\mathrm{pH})$ e condutividade elétrica do substrato (Cond).

Table 2. Development of the root system in Cattleya labiata and chemical characterization of the substrate. Dry root mass (MSR), average length of the main roots (CMR), number of roots (Raizes), pH of the substrate (pH) and electrical conductivity of the substrate (Cond).

\begin{tabular}{|c|c|c|c|c|c|c|c|c|c|c|}
\hline \multirow[b]{2}{*}{ Trat } & \multicolumn{10}{|c|}{ Sistema Radicular e Substrato } \\
\hline & $\begin{array}{l}\text { MSR } \\
\text { (mg) }\end{array}$ & & $\begin{array}{c}\text { CMR } \\
\text { (cm) }\end{array}$ & & $\begin{array}{c}\text { Raízes } \\
\left(\mathrm{n}^{\circ}\right)\end{array}$ & & pH & & $\begin{array}{c}\text { Cond } \\
\left(\mathrm{uS} \mathrm{c^{-1 } )}\right.\end{array}$ & \\
\hline Test & 1305 & $a^{1}$ & 13,7 & a & 23 & $\mathrm{~b}$ & 4,93 & $\mathrm{a}$ & 101 & $\mathrm{c}$ \\
\hline Peters & 1512 & a & 10,8 & $\mathrm{ab}$ & 27 & $\mathrm{ab}$ & 4,78 & $\mathrm{~b}$ & 180 & $\mathrm{~b}$ \\
\hline NPK & 1635 & a & 9,7 & $\mathrm{~b}$ & 34 & $\mathrm{a}$ & 4,80 & $a b$ & 249 & $\mathrm{a}$ \\
\hline NK & 1389 & a & 9,7 & $\mathrm{~b}$ & 31 & $\mathrm{ab}$ & 4,80 & $\mathrm{ab}$ & 163 & b \\
\hline $\mathrm{CV}(\%)$ & 27,41 & & 32,35 & & $15,21^{2}$ & & 2,91 & & 26,33 & \\
\hline
\end{tabular}

Médias seguidas da mesma letra não diferem pelo teste de Tukey a 5\%. Trat: tratamentos. Test: testemunha. Peters: Peters20 2020 . NPK: ureia + cloreto de potássio + superfosfato simples. NK: ureia + cloreto de potássio. ${ }^{2}$ Dados transformados $(\sqrt{\mathrm{x}}+1) .{ }^{l}$ Means followed by same letter don't differ by $5 \%$ Tukey test. Trat: treatments. Test: control. Peters: Peters 2020 20. NPK: urea + potassium chloride + superphosphate. NK: urea + potassium chloride.

${ }^{2}$ Transformed data $(\sqrt{ } x+1)$.

Em relação aos parâmetros químicos do substrato (Tabela 2), as maiores diferenças foram observadas na condutividade elétrica, que foi elevada pelas fertilizações, porém permaneceram abaixo de $500 \mu \mathrm{S} \mathrm{cm}^{-1}$, valor considerado como salinidade do substrato (TAKANE et al., 2010). Para o $\mathrm{pH}$ os tratamentos, Peters ${ }^{\circledR}$, NK e NPK agrícolas, não diferiram entre si, permanecendo dentro da faixa indicada para o cultivo de orquídeas em casca de pinus, que varia entre 3,5 a 5 (TAKANE et al., 2010).
Para os teores de nutrientes da parte aérea das orquídeas, foi possível observar que o $\mathrm{N}$, P e o $\mathrm{K}$ foram elevados nos tratamentos que receberam Peters ${ }^{\circledR}$ - NPK 202020 (3 $\left.\mathrm{g} \mathrm{L}^{-1}\right)$; NPK - ureia $\left(1,3 \mathrm{~g} \mathrm{~L}^{-1}\right)$, superfosfatos simples $\left(3,3 \mathrm{~g} \mathrm{~L}^{-1}\right)$ e cloreto de potássio $\left(1,0 \mathrm{~g} \mathrm{~L}^{-1}\right) ; \mathrm{NK}$ - ureia $\left(1,3 \mathrm{~g} \mathrm{~L}^{-1}\right)$ e de cloreto de potássio $\left(1,0 \mathrm{~g} \mathrm{~L}^{-1}\right)$ em relação à testemunha, contudo para o $\mathrm{Ca}$ o efeito foi inverso, e para o $\mathrm{Mg}$ os teores não foram alterados significativamente (Tabela 3).

Tabela 3. Teores de nutrientes da parte aérea em Cattleya labiata.

Table 3. Nutrients content in aerial part of Cattleya labiata.

\begin{tabular}{|c|c|c|c|c|c|c|c|c|c|c|}
\hline \multirow[b]{2}{*}{ Trat } & \multicolumn{10}{|c|}{ Nutrientes $\left(\mathrm{g} \mathrm{kg}^{-1}\right)$} \\
\hline & $\mathbf{N}$ & & $\mathbf{P}$ & & $\mathbf{K}$ & & $\mathrm{Ca}$ & & Mg & \\
\hline Test & 12,44 & $b^{1}$ & 3,22 & d & 21,71 & $\mathrm{c}$ & 28,21 & a & 3,30 & $\mathrm{a}$ \\
\hline Peters & 16,95 & $\mathrm{a}$ & 5,37 & a & 35,82 & $\mathrm{~b}$ & 22,99 & $\mathrm{~b}$ & 3,20 & $\mathrm{a}$ \\
\hline NPK & 18,56 & a & 4,86 & $b$ & 35,89 & $\mathrm{~b}$ & 23,19 & $\mathrm{~b}$ & 2,84 & a \\
\hline NK & 16,30 & a & 4,10 & $\mathrm{c}$ & 41,33 & a & 22,49 & $\mathrm{~b}$ & 3,24 & a \\
\hline $\mathrm{CV}(\%)$ & 12,60 & & 7,66 & & 14,66 & & 15,17 & & 14,05 & \\
\hline
\end{tabular}

${ }^{1}$ Médias seguidas da mesma letra não diferem pelo teste de Tukey a 5\%. Trat: tratamentos. Test: testemunha. Peters: Peters 202020 . NPK: ureia + cloreto de potássio + superfosfato simples. NK: ureia + cloreto de potássio. ${ }^{I}$ Means followed by same letter don't differ by 5\% Tukey test. Trat: treatments. Test: control. Peters: Peters 2020 20. NPK: urea + potassium chloride + superphosphate. NK: urea + potassium chloride. 
Para os teores de $\mathrm{N}$ as fertilizações com NPK, NK agrícolas e a comercial não diferiram entre si, contudo os teores de fósforo foram alterados pelos tratamentos, onde o maior teor de $\mathrm{P}$ foi observado no tratamento com Peters ${ }^{\circledR}$ e o menor no NK agrícola. Apesar dos menores teores de $\mathrm{P}$ no tratamento $\mathrm{NK}$, não foram observados sintomas de deficiência. Rodrigues, et al. (2010), estudando a fertilização em Laelia sp., observaram que os maiores teores de nutrientes na parte aérea foram de $\mathrm{K} \mathrm{e} \mathrm{N}$, sendo o P, o quarto ou o quinto maior teor, deste modo é possível inferir que durante o crescimento vegetativo o $\mathrm{K}$ e o $\mathrm{N}$ são os nutrientes mais demandados.

Apesar de importante no metabolismo energético e constituinte de membranas e ácidos nucléicos (MALAVOLTA, 2006), o P é exigido em menores concentrações, sendo indicadas relações N:P:K de 10:4:8 em Cattleya (NAIK et al., 2009), deste modo as quantidades de $\mathrm{P}$ disponibilizadas pelo substrato não foram limitantes para o crescimento inicial. Outro ponto relevante é que em sistemas naturais o $\mathrm{P}$ é absorvido rapidamente de soluções altamente diluídas e transitórias do dossel e, para plantas epífitas é o nutriente mais limitante. Deste modo as mesmas possuem alta eficiência na sua absorção bem como alta capacidade de realocação do nutriente (ZOTZ, 2004).

$\mathrm{O}$ maior teor de $\mathrm{P}$ no tratamento $\mathrm{NK}$ em relação à testemunha, uma vez que ambos os tratamentos não receberam o nutriente, ocorreu provavelmente pela maior mineralização da casca de pinus utilizada como substrato, no tratamento fertilizado com NK. Segundo Jackson et al. (2009), a adição de $300 \mathrm{mg} \mathrm{L}^{-1}$ de $\mathrm{N}$ em vasos de 1,7 $\mathrm{L}$ contendo casca de pinus aumentou a disponibilização de nutrientes como K e P. Em 14 semanas, os lixiviados apresentaram diferenças de 9,8 para $29,4 \mathrm{mg}$ P, e de 40 para $128,3 \mathrm{mg} \mathrm{K}$, entre os substratos que não receberam e os que receberam adição de $\mathrm{N}$. Os mesmos autores atribuem que o incremento da mineralização ocorre devido ao aumento na atividade biológica em resposta a adição de $\mathrm{N}$ sobre o substrato.

A redução nos teores de cálcio nos tratamentos Peters ${ }^{\circledR}$, NK e NPK agrícolas, ocorre devido ao maior crescimento das plantas nestes tratamentos, que resultou em um incremento em massa seca, não acompanhado pela absorção do nutriente em mesma intensidade, o que acarreta na diluição do mesmo nos tecidos. Este efeito é relatado em Laelia, (JIMÉNEZ-PEÑA et al., 2013), onde os autores destacam correlação positiva para N, P e K e negativa para $\mathrm{Ca}$ e $\mathrm{Mg}$, com o aumento da condutividade elétrica da solução nutritiva.Os teores tanto de Ca como $\mathrm{Mg}$ absorvidos, vieram provavelmente da água utilizada para a irrigação deste experimento, a qual por análise contém 50 $\mathrm{mg} \mathrm{L}^{-1}$ de Ca e $5 \mathrm{mg} \mathrm{L}^{-1}$ de $\mathrm{Mg}$.

Em relação ao acúmulo (Tabela 4) devido ao ganho em massa todos os tratamentos fertilizados foram superiores à testemunha para a maioria dos nutrientes, com exceção do Ca no tratamento com aplicação de Peters ${ }^{\circledR}$. Todavia quando comparadas as fertilizações entre si, os acúmulos foram similares, somente o acúmulo de fósforo no tratamento NK foi inferior ao Peters ${ }^{\circledR}$.

Tabela 4. Acúmulo de nutrientes da parte aérea em Cattleya labiata.

Table 4. Nutrients accumulation on aerial part of Cattleya labiata.

\begin{tabular}{|c|c|c|c|c|c|c|c|c|c|c|}
\hline \multirow[b]{2}{*}{ Trat } & \multicolumn{10}{|c|}{ Acúmulo de Nutrientes (mg vaso-1) } \\
\hline & $\mathbf{N}$ & & $\mathbf{P}$ & & $\mathbf{K}$ & & $\mathrm{Ca}$ & & Mg & \\
\hline Test & 17,47 & $c^{1}$ & 4,55 & $\mathrm{c}$ & 30,84 & $\mathrm{c}$ & 39,61 & $\mathrm{~b}$ & 4,68 & $\mathrm{~b}$ \\
\hline Peters & 40,00 & $\mathrm{a}$ & 12,64 & $\mathrm{a}$ & 84,71 & a & 54,22 & $a b$ & 7,51 & a \\
\hline NPK & 48,38 & a & 12,58 & $a b$ & 92,76 & a & 60,90 & a & 7,43 & a \\
\hline NK & 40,65 & a & 10,18 & $\mathrm{~b}$ & 104,05 & a & 55,76 & a & 8,13 & a \\
\hline $\mathrm{CV}(\%)$ & 26,60 & & 22,46 & & 28,05 & & 26,78 & & 27,04 & \\
\hline
\end{tabular}

${ }^{1}$ Médias seguidas da mesma letra não diferem pelo teste de Tukey a 5\%. Trat: tratamentos. Test: testemunha. Peters: Peters20 2020. NPK: ureia + cloreto de potássio + superfosfato simples. NK: ureia + cloreto de potássio. ${ }^{1}$ Means followed by same letter don't differ by 5\% Tukey test. Trat: treatments. Test: control. Peters: Peters 2020 20. NPK: urea + potassium chloride + superphosphate. NK: urea + potassium chloride.

A ordem dos elementos acumulados foram $\mathrm{K}>\mathrm{Ca}>\mathrm{N}>\mathrm{P}>\mathrm{Mg}$, entretanto quando não foi realizada fertilização essa ordem foi alterada sendo, $\mathrm{Ca}>\mathrm{K}>\mathrm{N}>\mathrm{Mg}>\mathrm{P}$. Essa alteração ocorreu pela disponibilidade de cálcio na água de irrigação e o não suprimento dos demais nutrientes.

Estes resultados sugerem que o uso das fontes agrícolas foram tão eficientes quanto o fertilizante solúvel comercial, pois o desenvolvimento inicial das plantas ocorreu de forma similar (Figura 1), sem alterar de forma prejudicial as relações entre os nutrientes. As fontes agrícolas de nutrientes podem ser uma alternativa de redução de custos em uma produção comercial de orquídeas. 


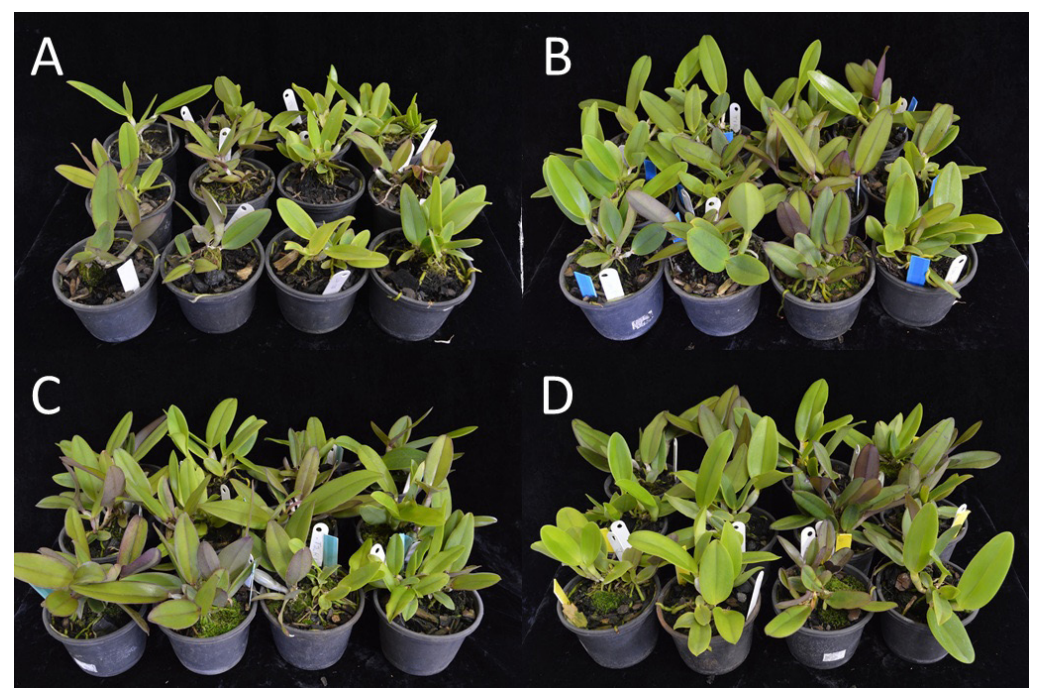

Figura 1. Desenvolvimento inicial de Cattleya labiata em diferentes fertilizações. A: sem fertilização (Testemunha). B: Peters20 20 20. C: ureia + cloreto de potássio + superfosfato simples (NPK). D: ureia + cloreto de potássio (NK).

Figure 1. Young development of Cattleya labiata under different fertilizations. A: without fertilization (Control). B: Peters 2020 20. C: urea + potassium chloride + superphosphate (NPK). D: urea + potassium chloride (NK).

\section{CONCLUSÃO}

O uso de ureia, superfosfato simples e cloreto de potássio, aplicados em solução nutritiva, apresentam potencial para substituir as formulações comerciais no crescimento inicial da orquídea nativa Cattleya labiata.

\section{REFERÊNCIAS}

BICHSEL, R.G.; STARMAN, T.W.; WANG, Y.T. Nitrogen, phosphorus, and potassium requirements for optimizing growth and flowering of the Dendrobium nobile as a potted orchid. HortScience, v.43, n.2, p.328-332, 2008.

CARDOSO, J.C.; MARTINELLI, A.P.; SILVA, J.A.T. A novel approach for the selection of Cattleya hybrids for precocious and season-independent flowering. Euphytica, v.210, p.143-150, 2016.

FERREIRA, D.F. Sisvar: um sistema computacional de análise estatística. Ciência e Agrotecnologia, v.35, n.6, p.1039-1042, 2011.

JACKSON, B.E.; WRIGHT, R.D.; ALLEY, M.M. Comparison of fertilizer nitrogen availability, nitrogen immobilization, substrate carbon dioxide efflux, and nutrient leaching in peat-lite, pine bark, and pine tree substrates. HortScience, v.44, n.3, p.781-790, 2009.

JIMÉNEZ-PEÑA， N.; VALDEZ-AGUILAR， L.A.; CASTILLO-GONZÁLES, A.M.; COLINAS-LEÓN, M.T.; CARMILL, A.D.; CARMILL, D.L. Growing media and nutrient solution concentration affect vegetative growth and nutrition of Laeliaanceps Lindl. HortScience, v.48, n.6, p.773-779, 2013.
JUNQUEIRA, A.H.; PEETZ, M.S. O setor produtivo de flores e plantas ornamentais do Brasil, no período de 2008 a 2013: atualizações, balanços e perspectivas. Revista Brasileira de Horticultura Ornamental, v.20, n.2, p.115$120,2014$.

KÄMPF, A.N.; TAKANE R.J.; SIQUEIRA, P.T.V. Floricultura; Técnicas de preparo de substratos. Brasília: LK, 2006. 132p.

MALAVOLTA, E. Manual de nutrição mineral de plantas. São Paulo: Agronômica Ceres, 2006. 638p.

MALAVOLTA, E.; VITTI, G.C.; OLIVEIRA, S.A. Avaliação do estado nutricional das plantas: princípios e aplicações. Piracicaba: POTAFOS, 1997. 319p.

NAIK, S.; BARMAN, D.; MEDHI, R.P. Evaluation of electrical conductivity of the fertilizer solution on growth and flowering of a Cymbidium hybrid. South African Journal of Plant and Soil, v.30, n.1, p.33-39, 2013.

NAIK, S.K.; USHA BHARATHI T.; BARMAN, D.; DEVADAS, R.; RAM, P.A.L.; MEDHI, R.P. Status of mineral nutrition of orchid-a review. Journal of Ornamental Horticulture, v.12, n.1, p.1-14, 2009.

NETO, A.E.F.; BOLDRIN, K.V.F.; MATTSON, N.S. Nutrition and quality in ornamental plants. Ornamental Horticulture, v.21, p.139-150, 2015.

NG, C.K.Y.; HEW, C.S. Orchid pseudobulbs false bulbs with a genuine importance in orchid growth and survival! Scientia Horticulturae, v.83, n.3, p.165-172, 2000. 
PAN, R.C.; YE, Q.S.; HEW, C.S. Physiology of Cymbidiumsinense: a review. Scientia Horticulturae, v.70, p.123-129, 1997.

RODRIGUES, D. T.; NOVAIS, R.F.; ALVAREZ, V.H.; DIAS, J.M.M.; VILLANI, E.M.A. Orchid growth and nutrition in response to mineral and organic fertilizers. Revista Brasileira de Ciências do Solo, v.34, n.5, p.16091616, 2010.

SUSILO, H.; PENG, Y.C.; LEE, S.C.; CHEN, Y.C.; CHANG, Y.C.A. The uptake and partitioning of nitrogen in Phalaenopsis Sogo Yukidian 'V3' as shown by $15 \mathrm{~N}$ as a tracer. Journal of the American Society for Horticultural Science, v.138, n.3, p.229-237, 2013.

TAKANE, R.J., YANAGISAWA, S.S., PIVETTA, K.F.L. Cultivo moderno de orquídeas: Cattleya e seus híbridos. Fortaleza: UFC, 2010.179 p.
TRÉPANIER, M.; LAMY, M.P.; DANSEREAU, B. Phalaenopsis can absorb urea directly through their roots. Plant and Soil, v.319, n.1-2, p.95-100, 2009.

WANG, Y.T.; KONOW, E.A. Fertilizer source and medium composition affect vegetative growth and mineral nutrition of a hybrid moth orchid. Journal of the American Society for Horticultural Science, v.127, n.3, p.442-447, 2002.

ZONG-MIN, M.; NING, Y.; SHU-YUN, L.; HONG, H. Nitrogen requirements for vegetative growth, flowering, seed production, and ramet growth of Paphiopedilum armeniacum (Orchid). HortScience, v.47, n.5, p.585-588, 2012.

ZOTZ, G. The resorption of phosphorus is greater than that of nitrogen in senescing leaves of vascular epiphytes from lowland Panama. Journal of Tropical Ecology, v.20, p.693-696, 2004. 